Supporting Information

\title{
3D Bioprinting of Bone Marrow Mesenchymal Stem Cell-laden Silk Fibroin Double Network Scaffolds for Cartilage Tissue Repair
}

Tianyu Ni, ${ }^{a, b}$ Min Liu, ${ }^{c}$ Yajie Zhang, ${ }^{a}{ }^{Y i} \mathrm{Cao}^{\mathrm{a}}$ and Renjun Pei*a

${ }^{a}$ CAS Key Laboratory for Nano-Bio Interface, Suzhou Institute of Nano-Tech and Nano-Bionics, Chinese Academy of Sciences, Suzhou, 215123, China

${ }^{b}$ Nano Science and Technology Institute, University of Science and Technology of China, Suzhou 215123, China.

${ }^{\mathrm{c}}$ Institute for Interdisciplinary Research, Jianghan University, Wuhan 430056, China 


\section{Synthesis and characterization of HPMC-MA}

The characteristic peak of $\delta_{-\mathrm{CH}=\mathrm{CH}-}(\delta \sim 5.5-6.5)$ and $\delta_{-\mathrm{COCH} 3}(\delta \sim 1.9)$ indicated that MA was successfully grafted on the HPMC. Besides, with increasing the dosage of MA, the strength of the characteristic peak was also increased.

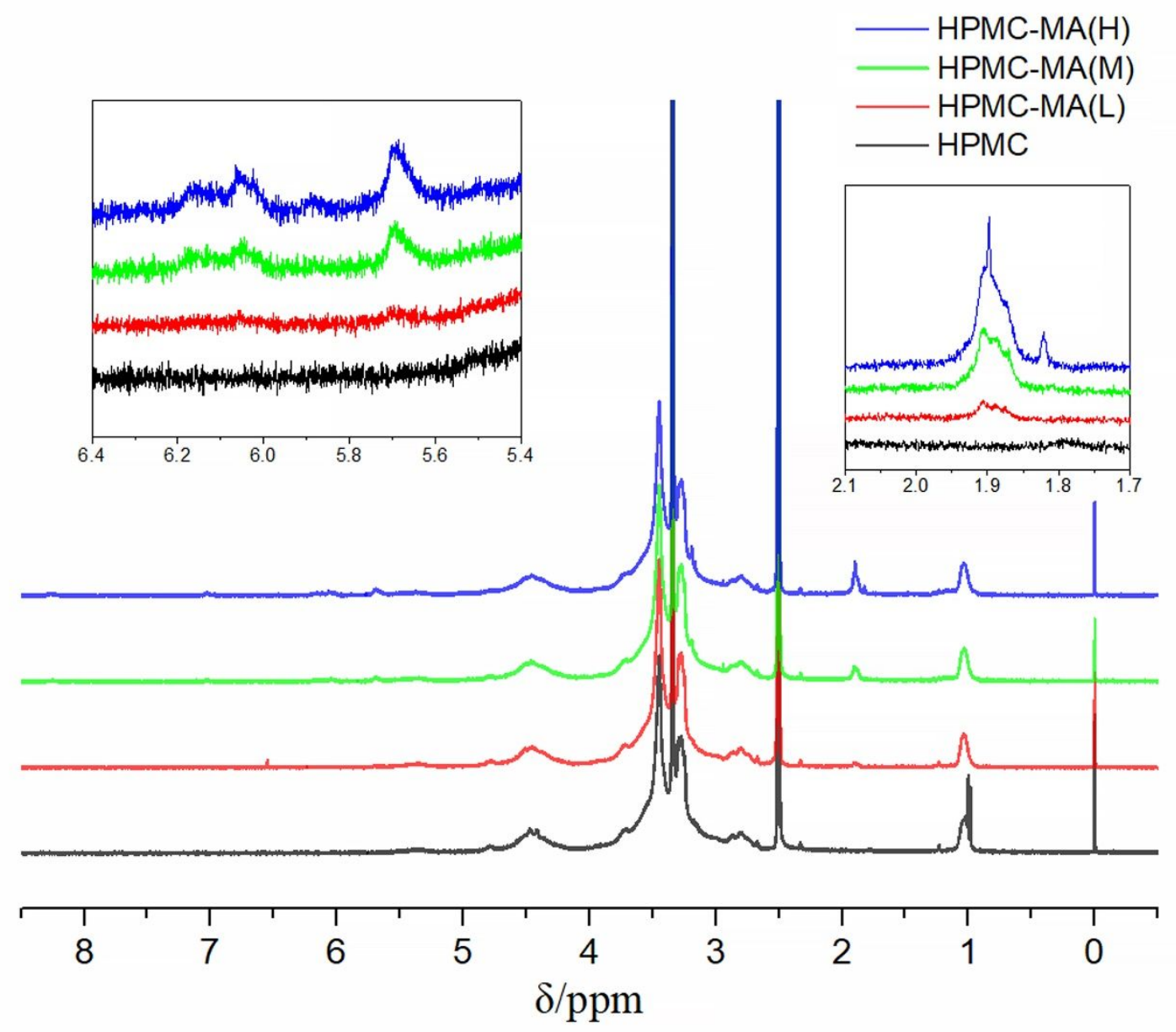

Figure S1. ${ }^{1} \mathrm{H}$ NMR spectrum of HPMC and HPMC-MA (recorded in DMSO-d ${ }_{6}$ ) 


\section{Cyclic compressive performance of SF/HPMC or SF/HPMC-MA hydrogels}

We investigated the effects of different MA contents on mechanical properties of SF/HPMC-MA hydrogels. At larger strain, the SF/HPMC, SF/HPMC-MA(M) and $\mathrm{SF} / \mathrm{HPMC}-\mathrm{MA}(\mathrm{H})$ hydrogels fractured, thus showing a decrease in stress on the curve. While SF/HPMC-MA(L) hydrogel showed the greatest compressive strength and reproducibility.
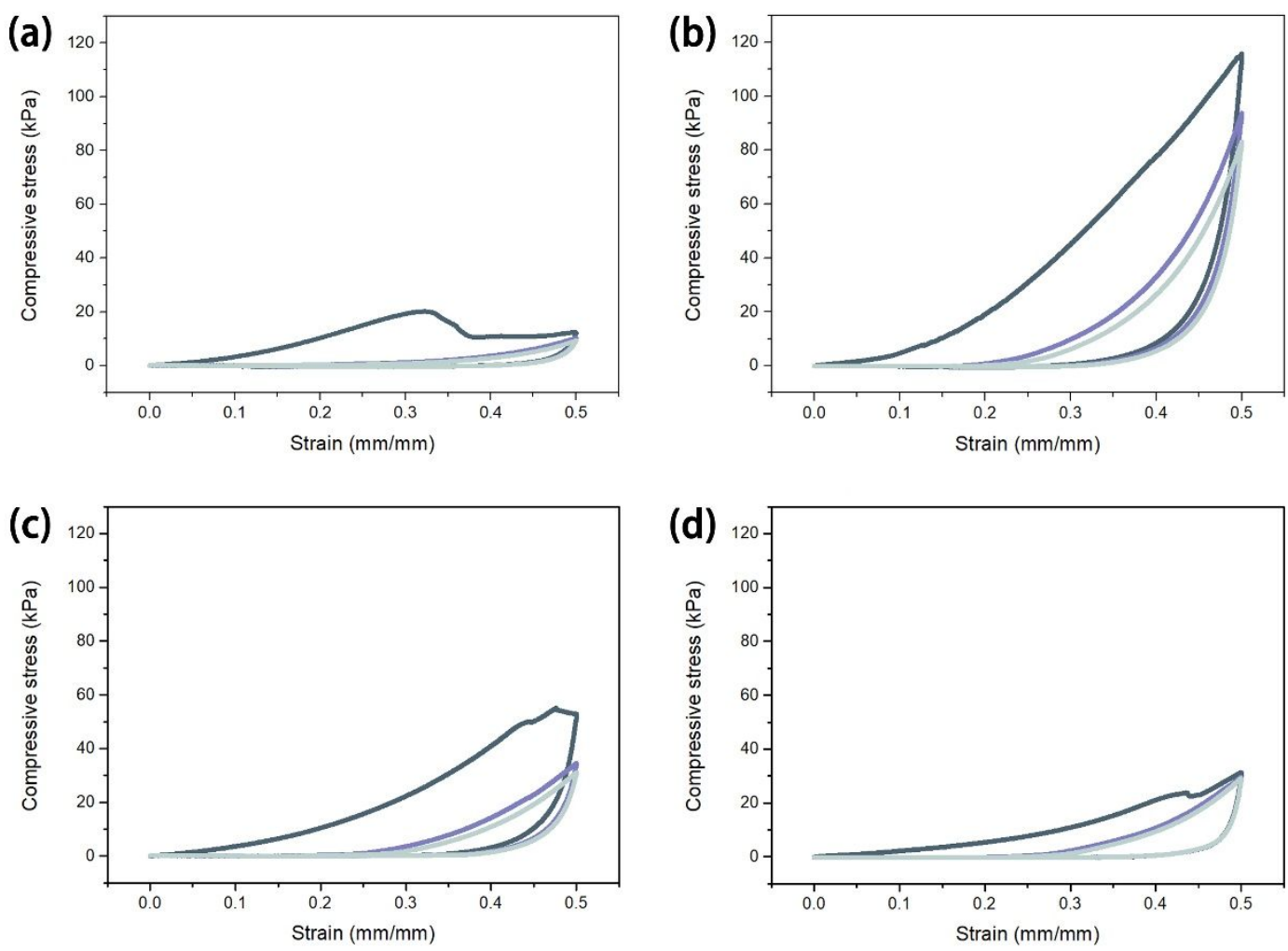

Figure S2. The effects of different MA contents on mechanical properties of SF/HPMC or SF/HPMC-MA hydrogels were shown in stress-strain curves with the first three cyclic compressive, a) SF/HPMC, b) SF/HPMC-MA(L), c) SF/HPMC-MA(M), d) SF/HPMC-MA(H). The solid content of each sample was 4 $\mathrm{wt} \%(3 \mathrm{wt} \% \mathrm{SF}+1 \mathrm{wt} \%$ HPMC or HPMC-MA) 


\section{The elastic shear modulus of SF/HPMC-MA(L)1 hydrogel}

The G' of SF solution after ultrasonication was no obvious difference at $2 \mathrm{~h}$ or $12 \mathrm{~h}$, indicated the self-assembly of SF nanofibrils was finished almost within $2 \mathrm{~h}$.
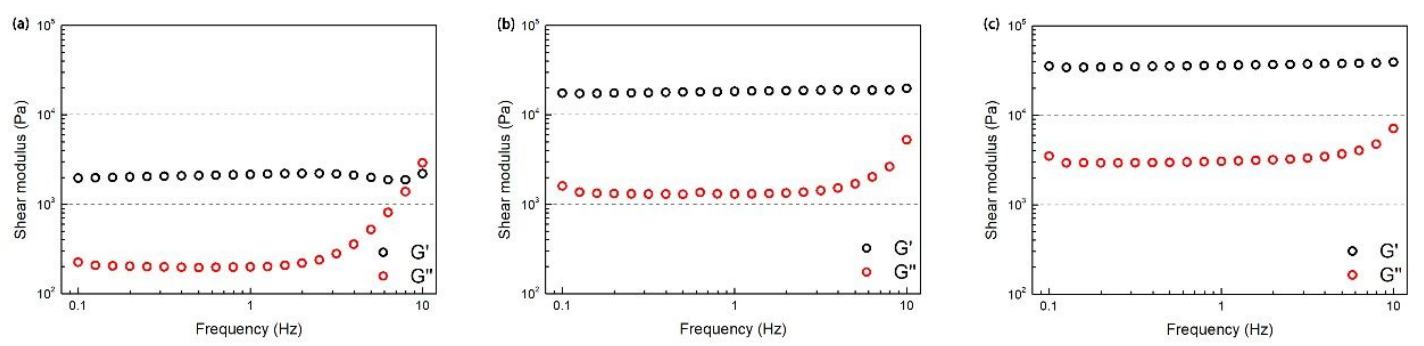

Figure S3. The shear modulus (G' \& G'') of SF/HPMC-MA(L)1 hydrogel after ultrasonication (with UV light) at $37{ }^{\circ} \mathrm{C}$, at frequency of $0.1-10 \mathrm{~Hz}$. The solid content was a) $2 \mathrm{wt} \%$, b) $4 \mathrm{wt} \%$ and c) $6 \mathrm{wt} \%$.

\section{Raman spectra of freeze-dried samples at Amide III region}

According to literatures, $1229 \mathrm{~cm}^{-1}$ was associated to $\beta$-sheet conformations of SF, while the peaks around 1245, 1252, 1264 and $1276 \mathrm{~cm}^{-1}$ were about random coil and disordered configuration of Amide III Silk I configuration ${ }^{1}$. The conclusion was consistent with the Amide I region observation. 


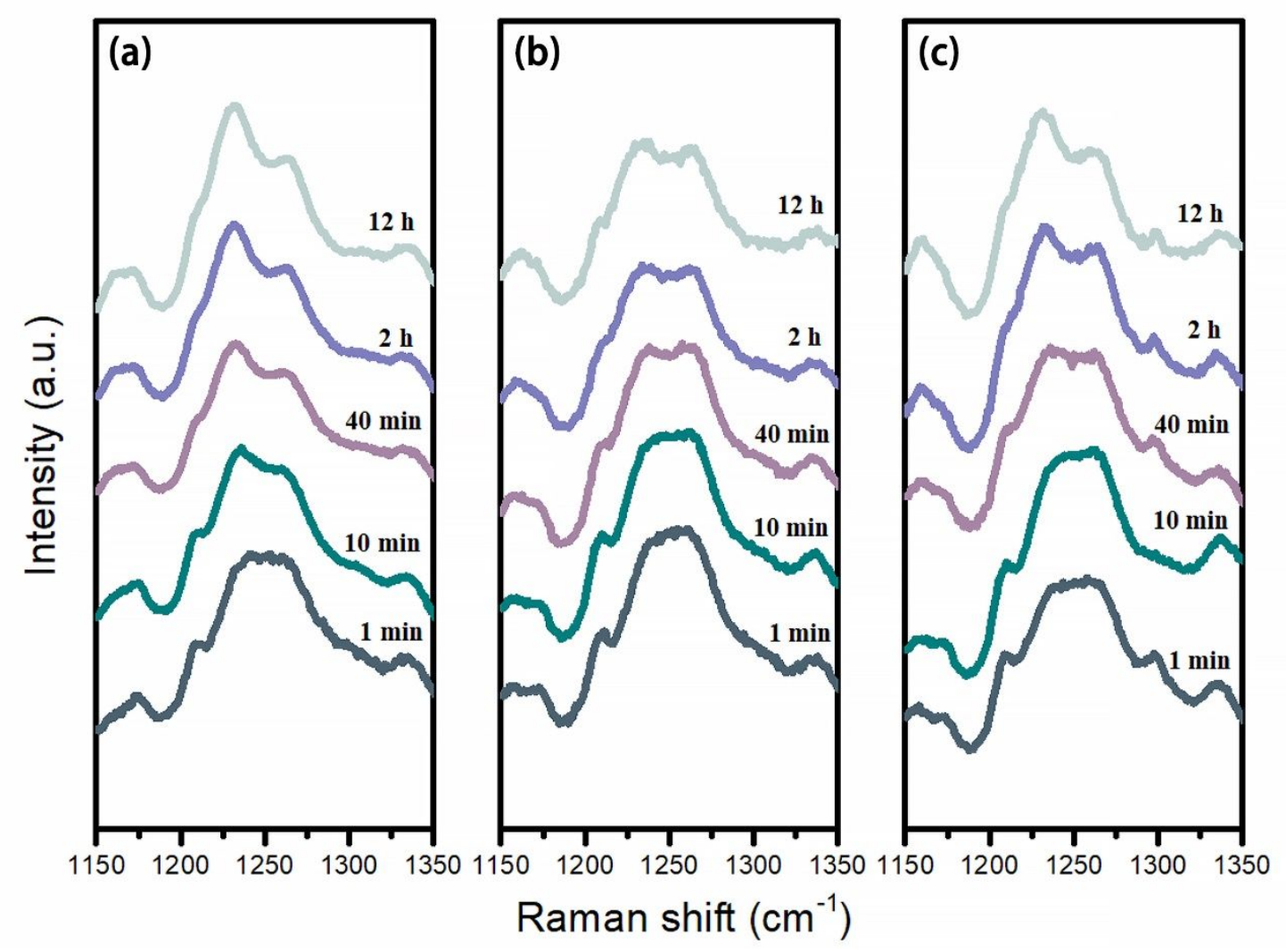

Figure S4. Amide III region of Raman spectra of freeze-dried samples incubated at $37{ }^{\circ} \mathrm{C}$ for $1,10,40,120$ and $720 \mathrm{~min}$, respectively; a) $\mathrm{SF}$ solutions; b) SF/HPMC-MA(L)1 prepolymer; SF/HPMC-MA(L)1 hydrogel with UV light. All mixtures were of $4 \mathrm{wt} \%$.

\section{The LCST of HPMC and HPMC-MA}

The rheological properties of HPMC and HPMC-MA were typical LCST behavior. The gel point defined as elastic modulus (G') needed to reach $100 \mathrm{~Pa}$, which were found that the cellulose's LCST decreased with increasing the grafting ratio of MA group (from $62^{\circ} \mathrm{C}$ to $47^{\circ} \mathrm{C}$ ). 

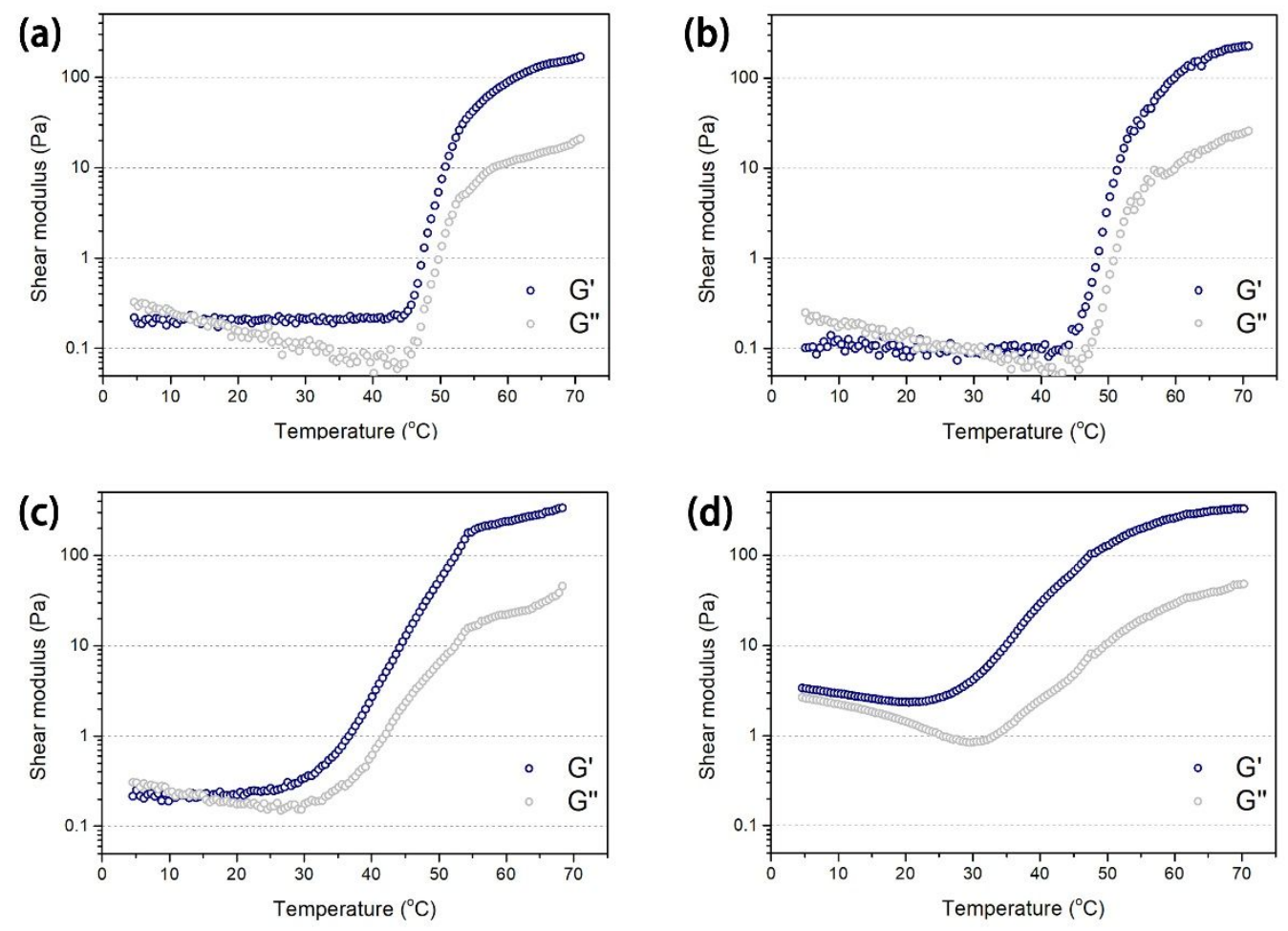

Figure S5. Temperature evolution of shear modulus ( $\left.G^{\prime} \& G^{\prime \prime}\right)$ of $1 \mathrm{wt} \%$ cellulose solution heating from $5{ }^{\circ} \mathrm{C}$ to $70{ }^{\circ} \mathrm{C}$, a) HPMC, b) HPMC-MA(L), c) HPMC-MA(M), d) HPMC-MA(H).

The process of fibrillar network of SF in SF and SF/HPMC-MA(L)1
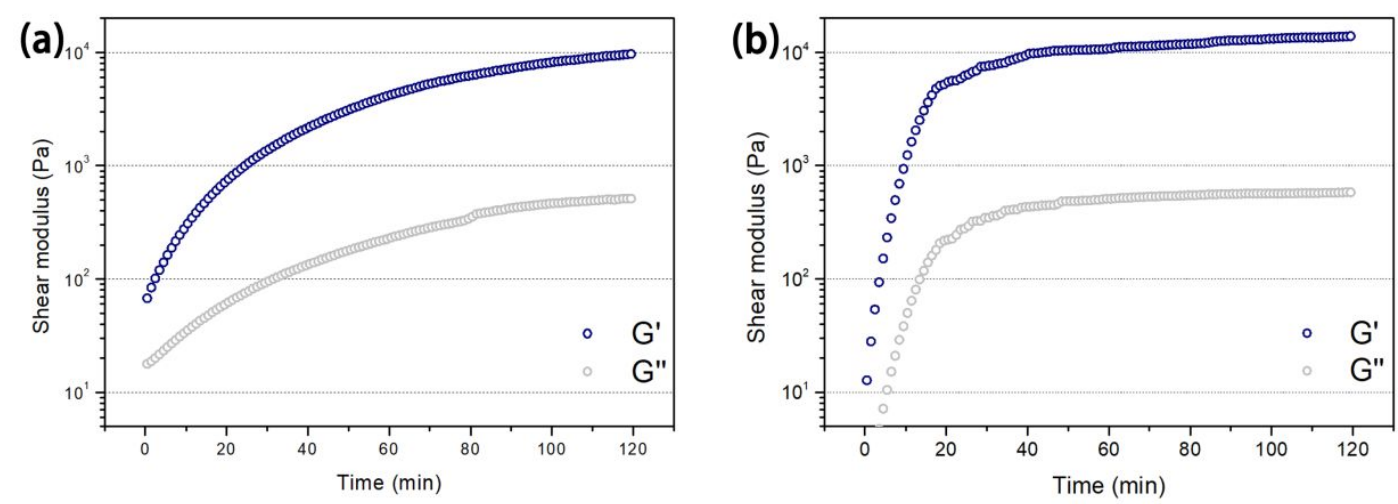

Figure S6. Time evolution of shear modulus ( $\left.G^{\prime} \& G^{\prime \prime}\right)$ of SF and SF/HPMC-MA(L)1 pre-solution after ultrasonication (without UV light) at $37^{\circ} \mathrm{C}$, a) 
SF, b) SF/HPMC-MA(L)1.

\section{References}

1. Monti, P.; Taddei, P.; Freddi, G.; Asakura, T.; Tsukada, M., Raman spectroscopic characterization of Bombyx mori silk fibroin: Raman spectrum of Silk I. Journal of Raman Spectroscopy 2001, 32 (2), 103-107. 